\title{
A HARDWARE TRANSVERSE BEAM FREQUENCY RESPONSE SIMULATOR*
}

\author{
J. Ning and C.Y. Tan ${ }^{\#}$, FNAL, Batavia, IL 60510, U.S.A.
}

\begin{abstract}
We built an electronic instrument that can mimic the transverse beam frequency response. The instrument consists of 1) a time delay circuit with an analog-todigital converter (ADC) which contains a first-in-first-out random assess memory (FIFO RAM) and a digital-toanalog converter (DAC); 2) a variable phase shifter circuit which is based on an all pass filter with a bandwidth of $25 \mathrm{kHz}$ to $30 \mathrm{kHz}$ and 3) a commutating filter which is a nonlinear band pass filter. With this instrument, we can dynamically adjust the betatron tune, the synchrotron tune, and the chromaticity. Using this instrument, we are able to test other beam systems without using actual beam.
\end{abstract}

\section{INTRODUCTION}

The Tevatron is an operational machine with limited time for machine studies. In order to save precious study time to test projects like the Tevatron transverse narrow band damper system and the phase locked loop tune tracker system, we built an instrument which simulates the Tevatron transverse beam frequency response. This instrument has a frequency response that is close to the measured Tevatron transverse beam frequency response (see Figure 1).

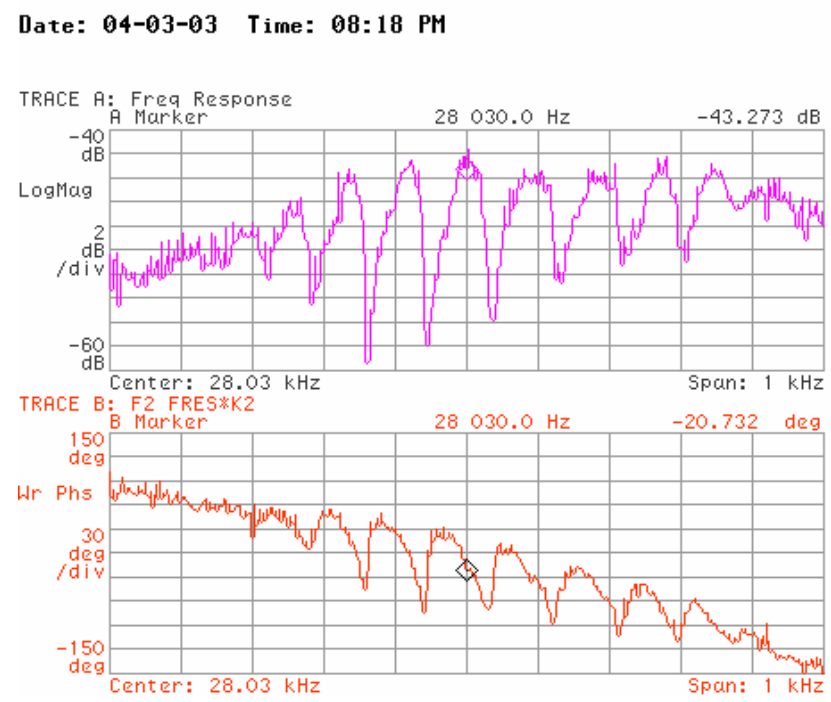

Figure 1. The measured Tevatron transverse beam frequency response.

There are three important parameters in the frequency response. The measured center frequency which is the betatron tune $(28.030 \mathrm{kHz}$ in Figure 1); the frequency

*Work supported by the US-LARP collaboration and by the Universities Research Association Inc. under Contract No. DE-AC0276CH03000 with the United States Department of Energy.

"cytan@fnal.gov distance between the peaks of the magnitude response, which is the synchrotron tune $(\sim 84 \mathrm{~Hz}$ in Figure 1$)$ and the envelope of the magnitude response which comes from chromaticity.

This electronic black box is used to mimic the measured frequency response shown in Figure 1. Also, the simulator is able to dynamically change the three parameters of the frequency response discussed above.

\section{THE SOLUTION}

Our approach in the design of the black box design is shown in Figure 2 and can be described as follows:

- Commutating filter. The filter's frequency response looks like that of coasting beam, which is without synchrotron sidebands.

- Time delay element. This is in the feedback loop to create the synchrotron sidebands in the frequency response of the commutating filter.

- Phase shifter. This is in the feedback loop to smoothly shift the synchrotron sidebands.

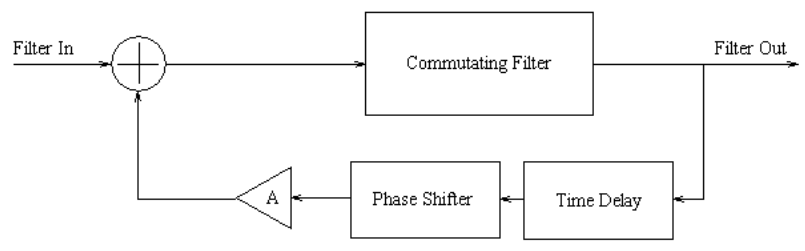

Figure 2. The block diagram of the hardware transverse beam frequency response simulator.

\section{Commutating Filter}

A commutating filter is an $R C$ low pass filter with several grounded capacitors [2]. A switch sequentially swaps one capacitor to another (see Figure 3).

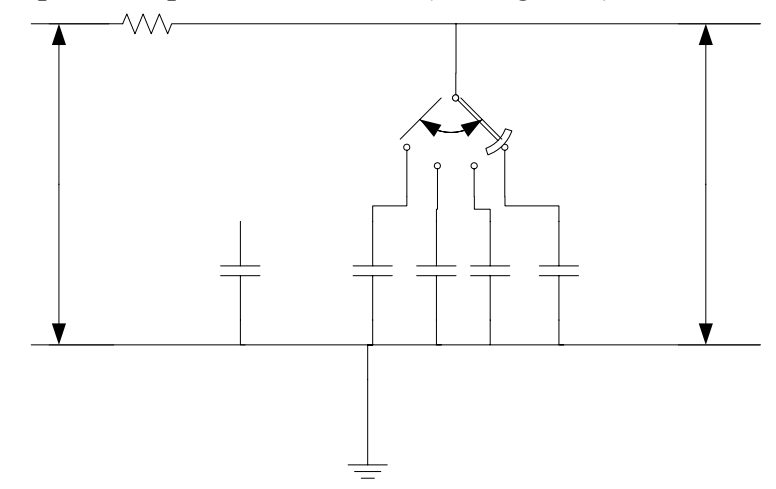

Figure 3. The commutating filter 
The switch swaps $C_{1}$ to $C_{N}$ sequentially with frequency $\omega_{S}$; this implies that the switch stops at each capacitor for $2 \pi / \omega_{S}$ and stops at the next capacitor for another $2 \pi / \omega_{S}$, and so on. We define $T_{S}=2 \pi N / \omega_{S}$. It takes $T_{S}$ for the switch to sample from $C_{1}$ to $C_{n}$. The transfer function of the commutating filter [3] can be expressed as

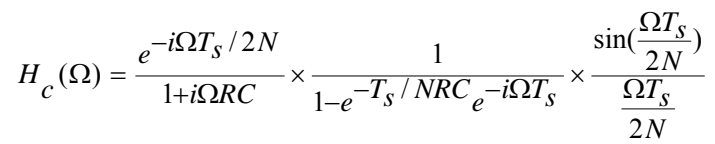

$$
=\text { phase shift } \times \text { resonance } \times \text { rectangle sample }
$$

For example, if $N=8, R=1 \mathrm{k} \Omega, C=0.1 \mu \mathrm{F}, T_{S}=1 / 25 \mathrm{kHz}$, we can plot the frequency response of the commutating filter around $25 \mathrm{kHz}$ as shown in Figure 4 and Figure 5.

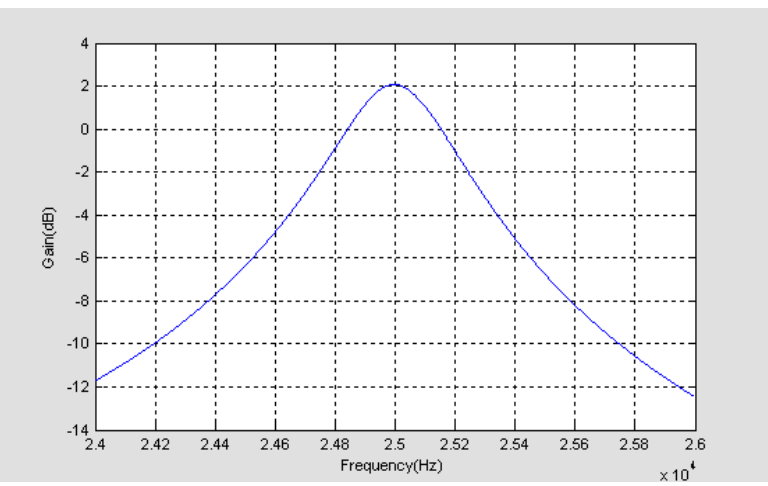

Figure 4. The magnitude response from Equation 1. It is close to the transverse beam magnitude response without synchrotron sidebands. The peak position is the betatron tune and is determined by $1 / T_{S}$. The peak can be dynamically changed by changing the switching frequency of the commutating filter.

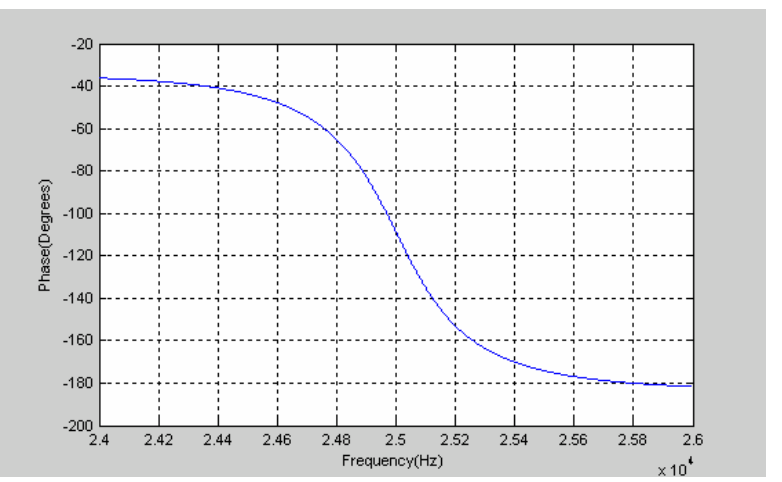

Figure 5. The phase response from Equation 1. It is close to the transverse beam phase response without the synchrotron sidebands.

The $-3 \mathrm{~dB}$ bandwidth of the commutating filter can be shown to be $1 / \pi N R C$.

\section{Time Delay Element}

A time delay element is required in the feedback loop shown in Figure 2 to make the synchrotron sidebands. The signal is sampled by an ADC and stored into two parallel FIFO RAMs, each with a memory depth $m$. The output of the FIFO RAMs are connected to a DAC. The clock signals of the ADC, FIFO RAMs and DAC are driven synchronously by a common clock $f_{C}$. The total time delay is given by Equation 2 .

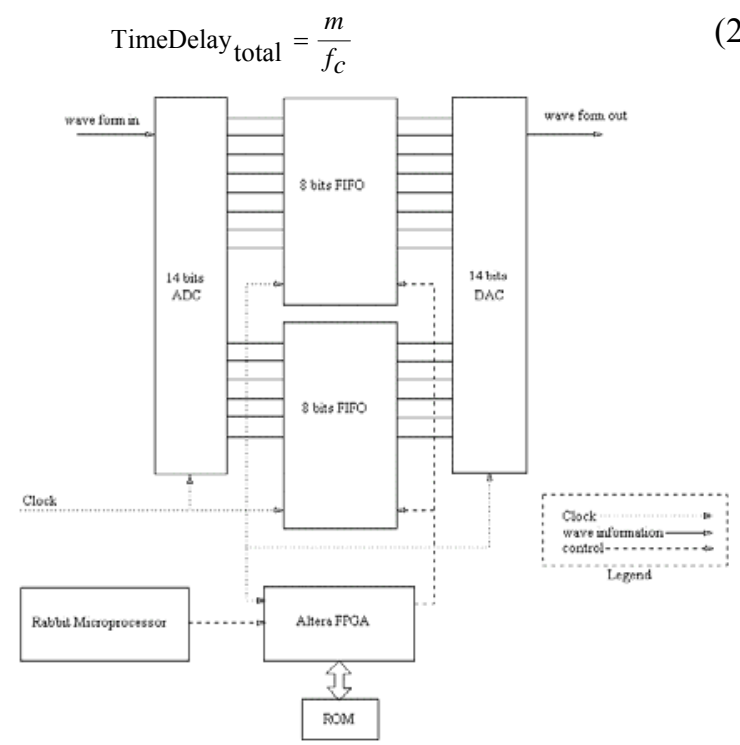

Figure 6. The block diagram of the digital time delay element. The ADC, FIFO RAMs and DAC are driven by a common clock. We can adjust the time delay by adjusting the period of the common clock.

By putting the digital time delay element with delay $\Delta t$ and a gain of $A$ into the feedback loop, we get the following transfer function

$$
H(\Omega)=\frac{H_{C}(\Omega)}{1+A H_{C}(\Omega) e^{-i \Omega \Delta t}}
$$

$|H(\Omega)|$ is plotted in Figure 7.

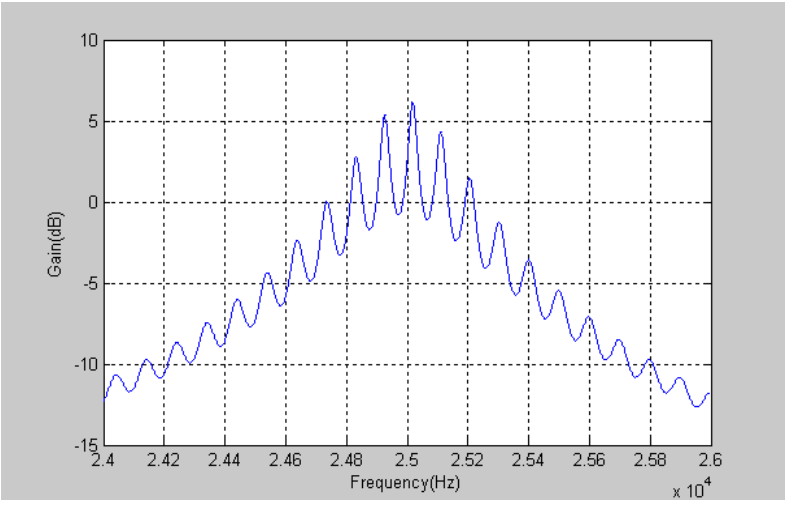

Figure 7. The magnitude response of a commutating filter with $0.01 \mathrm{~s}$ time delay and a gain of 0.3 in the feedback loop. The synchrotron sidebands created here are spaced approximately by $1 / \Delta t$ (see Figure 8 ). The envelope of the response (or chromaticity) is controlled by $1 / \pi N R C$. 


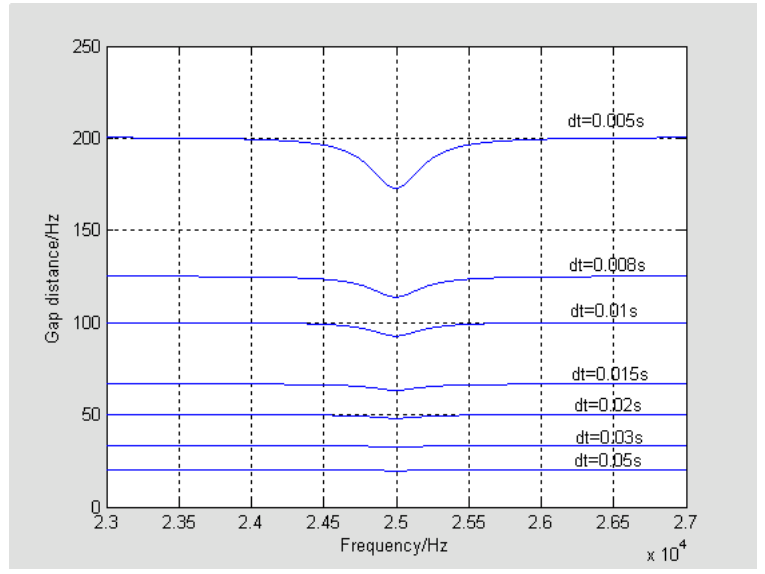

Figure 8 . The control of the gap distance

\section{Phase Shifter}

An arbitrary phase shifter in the feedback loop is used to smoothly move the synchrotron sidebands structure when the center frequency or betatron tune is moved. The block diagram of the phase shifter is shown in Figure 9. The phase shifter is set up to have best linearity in the frequency range $25-30 \mathrm{kHz}$.

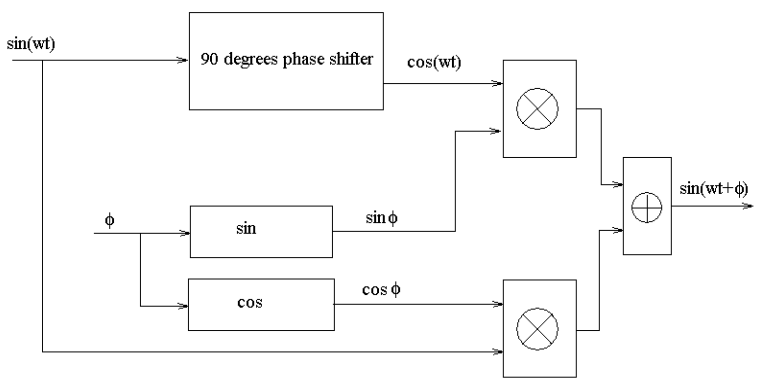

Figure 9. An arbitrary degrees phase shifter.

\section{MEASUREMENT}

Based on the approach described in the previous section, the measured frequency response is shown in Figure 10. The two traces shown here have the same time delay, and $R C$ time constant. This means that the synchrotron frequency and chromaticity are the same in both cases. The center frequency or betatron tune is different but the overall synchrotron sideband structure is nearly the same. The small difference in the frequency response comes from the non-linearity of the 90 degrees phase shifter.
Date: 04-29-03 Time: 11:54 AM

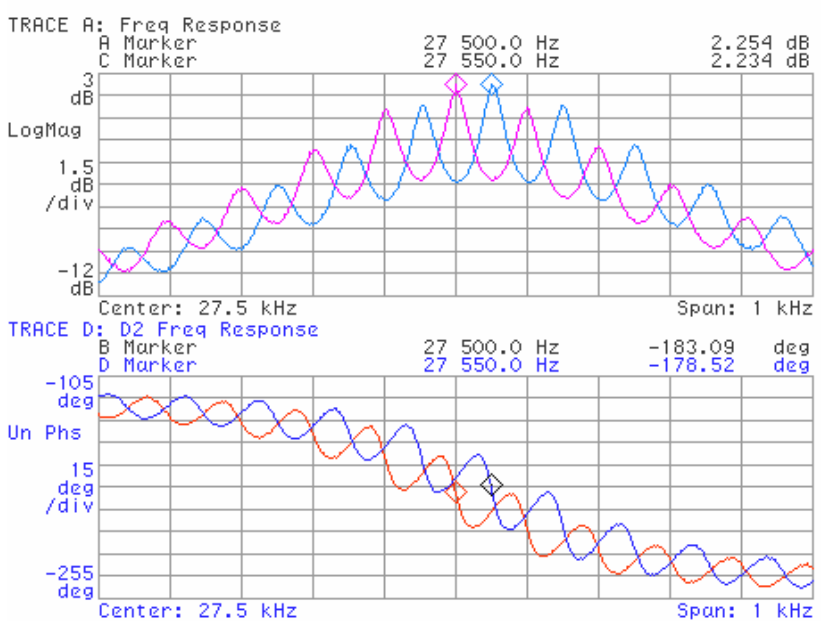

Figure 10. Two measurements of the hardware transverse beam frequency response simulator with two different center frequencies or betatron tunes. The phase responses are not perfectly shifted because of the non-linearity of the 90 degrees phase shifter.

\section{CONCLUSION}

We have described an approach to simulate the frequency response of the transverse beam frequency response based on a commutating filter. The characteristics of the commutating filter are studied. Two elements have been applied in the feedback loop of the commutating filter to obtain a result that we expected. The whole circuit is able to simulate the transverse beam frequency response. Three parameters of the transverse beam frequency response can also be changed dynamically.

In the future, an improved 90 degrees phase shifter with better linearity will improve the frequency response of the simulator. Two DDS (direct-digital-synthesis) chips will be added to the circuits to provide programmable onboard clock sources. The board can also be connected to a computer with Ethernet to gain remote control of the simulator.

\section{REFERENCES}

[1] J. Ning, "A Hardware Tevatron Transverse Beam Frequency Response Simulator", M.S. thesis 2005

[2] D. Peterson, Private Communication, 2003.

[3] C.Y. Tan, "Commutating Filter", unpublished, 2004. 